\title{
Review of ${ }^{90}$ Y-ibritumomab tiuxetan as first-line consolidation radio-immunotherapy for B-cell follicular non-Hodgkin's lymphoma
}

This article was published in the following Dove Press journal:

Cancer Management and Research

20 October 2009

Number of times this article has been viewed

\section{Christos Emmanouilides \\ Department of Medical Oncology, Interbalkan Hospital, Thessaloniki, Greece}

Correspondence: Christos Emmanouilides Department of Medical Oncology, Interbalkan Hospital, I0 Asklipiou St, 5700 I Pylaia,Thessaloniki, Greece

Tel +30 2310 400849

Fax +30 23I0 40049I

Email chrem@interbalkan-hosp.gr

\begin{abstract}
Several studies have indicated that radioimmunotherapy is an effective and clinically relevant complementary therapeutic approach for patients with B-cell non-Hodgkin's lymphoma (NHL) and may convert partial to complete response when given as consolidation after induction chemotherapy. Yttrium-90( $\left.{ }^{90} \mathrm{Y}\right)$-ibritumomab tiuxetan $\left({ }^{90} \mathrm{Y}-\mathrm{IT}, \mathrm{Zevalin}{ }^{\circledR}, \mathrm{Y} 2 \mathrm{~B} 8\right)$ has documented efficacy for both indolent and aggressive NHL. Patients considered eligible for ${ }^{90} \mathrm{Y}$-IT treatment should satisfy several screening criteria. A recently completed randomized study for patients with follicular lymphoma has demonstrated that ${ }^{90} \mathrm{Y}$-ibritumomab consolidation also produced a marked prolongation of the median time to progression from 13.5 to 37 months, while partial responders seem to derive relatively more benefit. Other published and ongoing studies explore a similar use for patients with aggressive lymphoma. Studies are comparing the use of ${ }^{90} \mathrm{Y}$-IT consolidation with the anti-CD20 antibody rituximab maintenance, which is also gaining acceptance. In conclusion, the documented benefit of radioimmunotherapy should be viewed in the context of the goals of treatment and the changing standards of care for lymphoma.
\end{abstract}

Keywords: radioimmunotherapy, ${ }^{90} \mathrm{Y}$-ibritumomab tiuxetan, follicular lymphoma, consolidation

\section{Introduction}

The therapeutic application of monoclonal antibodies has revolutionized the treatment of lymphoma. ${ }^{1}$ Although engagement and direct interaction with surface proteins appear to be of therapeutic value, antibodies also constitute excellent targeting systems, marking selected cells for the interaction with innate immune effector mechanisms, or by conjugation with moieties of therapeutic value which are thus locally delivered. Because radioimmunotherapy (RIT) has been shown to be sufficiently effective, the US Food and Drug Administration approved the first ever radioimmunoconjugate (RIC) for the treatment of a malignancy in February of 2002, when ${ }^{90} \mathrm{Y}$-ibritumomab tiuxetan $\left({ }^{90} \mathrm{Y}\right.$-IT; Zevalin ${ }^{\circledR}$; RIT Oncology) was licensed for the treatment of indolent or transformed, relapsed or refractory B-cell lymphoma. Soon therafter, a second anti-CD20 radioconjugate ${ }^{131} \mathrm{I}$ tositumomab (Bexxar ${ }^{\circledR}$; GlaxoSmithKline) was also approved. ${ }^{2,3}$ In Europe, ${ }^{90} \mathrm{Y}$-IT was subsequetly approved for the treatment of follicular lymphoma, relapsed or refractory after prior rituximab-containing treatment. Ibritumomab (IDEC-2B8) is the murine anti-CD20 antibody developed by Biogen-IDEC (San Diego, CA, USA); its chimeric version, rituximab (Rituxan ${ }^{\circledR}$; Genentech; Mabthera ${ }^{\circledR}$; F. Hoffmann-La Roche Ltd), is also available and widely used. Tiuxetan connotes the linker used (MxDTPA) to conjugate ${ }^{90} \mathrm{Y}$ to the antibody. 
RIT offers several advantages over external beam irradiation. Normal tissues overlying the tumor mass are prevented from significant radiation exposure. Since the RIC is given intravenously, it provides systemic radiation treatment to known as well as unsuspected tumor cells. It should be noted that neither rituximab nor the RIC available or under development are truly tumor specific as they bind to a marker also present at the normal lymphocyte counterparts. However, mounting experience from trials and clinical practice suggests that prolonged normal B-cell depletion is not associated with significant sequelae. ${ }^{4}$ As a pure $\beta$ emitter, ${ }^{90} \mathrm{Y}$ seems to offer theoretical and practical advantages, mainly simple handling practices. ${ }^{5,6}$ The lack of $\gamma$ component simplifies radioprotection during handling and administration of the RIC.

\section{${ }^{90} \mathrm{Y}-\mathrm{IT}$ for the treatment of lymphoma}

During the registrational clinical trials, a meticulous and thorough dosimetry procedure was performed, largely using data derived from indium-111 ( ${ }^{111}$ In-IT) kinetics. ${ }^{7,8}$ The purpose of dosimetry was to eliminate the possibility of excessive radiation exposure of vital organs. Based on favorable observations on kinetics and safety, the requirement of imaging has been eliminated in most countries. ${ }^{9}$

Patients considered eligible for ${ }^{90} \mathrm{Y}$-IT treatment should satisfy several screening criteria, including having less than $25 \%$ involvement of the bone marrow by disease determined by adequate core biopsy, absence of myelodysplasia, and satisfactory blood counts. In the phase I/II study, it became apparent that the major toxicity was reversible myelosuppression with median absolute neutrophil count (ANC) $1100 / \mu \mathrm{L}$ and median nadir thrombocytopenia of $49,500 / \mu \mathrm{L} .{ }^{10}$ The extent of bone marrow involvement by lymphoma and the pre-treatment platelet counts correlate with the risk of myelosuppression. The remainder of the toxicity was mostly associated with the infusion of rituximab, with the possible exception of mild fatigue and mild nausea.

Studies of RIT as singe agent indicate that the expected response rate for patients fulfilling the above criteria with relapsed or refractory indolent or transformed B-cell NHL is $74 \%$ to $80 \%$ and duration of response is approximately 1 year. ${ }^{4,11-13}$ Particularly longer remissions for several years were observed in subsets of patients. ${ }^{4}$ Importantly, in the 211 patients of the registrational studies, there was no difference in toxicity and efficacy of the treatment among the 113 patients less than 60 years old, the 58 patients of the age group between 60 and 70 and the 40 patients over $70 .{ }^{14}$ For aggressive B-cell lymphoma, the activity of ${ }^{90} \mathrm{Y}$-IT has been documented in the phase I/II study ${ }^{10}$ and it was confirmed in a multicenter prospective phase II study involving 102 evaluable patients, ${ }^{15}$ in the latter, an overall $44 \%$ response rate with $27 \%$ complete response $\mathrm{CR}$ ) rate was observed. The results were more favorable in rituximab naïve patients. In relapsed mantle cell lymphomas the activity of ${ }^{90} \mathrm{Y}$-IT has been reported in the range of $30 \%$ to $40 \%$ but responses usually last a few months. ${ }^{16}$

\section{Rationale for consolidation use of RIT}

There are many reasons to consider the delivery of radiotherapy immediately after a course of chemotherapy, ie, as commonly referred to, as consolidation treatment. It is conceivable that induction chemotherapy may "prime" patients for an enhanced RIT effect. In multivariate analysis of 203 patients involved in the ${ }^{90} \mathrm{Y}$-IT registrational studies, a factor that predicted for better response was tumor bulk; ${ }^{16}$ patients with nodal masses more than $5 \mathrm{~cm}$ had a $68 \%$ response rate, as opposed to $90 \%$ for patients with smaller tumors $(P<0.001)$. Thus, cytoreduction achieved by chemotherapy may render patients more amenable to RIT. In addition, chemotherapy treatment usually results in amelioration of bone marrow disease, so that the likelihood of considerable bone marrow involvement exceeding $25 \%$ is extremely low. This may translate in reduced bone marrow exposure to irradiation. Furthermore, the impact of RIT on measurable residual disease could be quantified, ie, one could know how many partial responses could be converted to complete ones.

Retrospective meta-analysis of the registrational trials indicate a considerably better outcome in patients treated with radiotherapy early in their disease course ${ }^{17}$ for instance, in patients with follicular lymphoma receiving ${ }^{90} \mathrm{Y}$-IT as second line treatment, the complete and overall response rate were $51 \%$ and $89 \%$ respectively. The complete response (CR) rate and time to progression (TTP) were statistically better compared to the group of patients with more prior treatments $(P<0.05)$. Patients who achieved a CR had a median 2-year TTP. Such data suggest that it may be preferable to move up ${ }^{90} \mathrm{Y}$-IT treatment in the sequence of therapy for lymphoma, in order to take advantage of the high likelihood of achieving a CR, which confers a longer TTP. In fact, a response rate of $100 \%$ has been reported after first-line use of RIT in patients with follicular lymphoma. ${ }^{18}$ Late toxicity and particularly myelodysplasia seems not to be an issue, so that subsequent treatments upon relapse are possible. ${ }^{19,20}$ 
All the above observations support the use of RIT as a practical and convenient consolidation treatment after induction chemotherapy as it may be associated with increased efficacy and lack of long-term toxicity.

Nevertheless, there are certain theoretical concerns for indiscrete consolidation treatment with RIT. The ratio of beneficial radiation vs radiation deposited to surrounding tissue depends on the size of the lymphomatous mass and the path length of the radioisotope used. Since most of the radiation energy is delivered within a sphere with a radius of a few millimeters, if one conceptualizes residual disease as a dispersion of single cells, most of the radiation emitted by the lymphoma-attached radioconjugate would be wasted. On the other hand, the definition of complete response is a convention, which almost certainly includes patients with small lymphomatous aggregates in "normal size" lymph nodes of less than $1.5 \mathrm{~cm}$. Thus, complete responders may still derive benefit from the crossfire effect. This is more valid if the response to preceding chemotherapy was not complete; in such cases, RIT may be an ideal agent to treat the remaining involved nodes. Chemotherapy-resistant lymphoma cells may still be susceptible to radiation as significant activity of RIT in patients with chemotherapy refractoriness has been reported. ${ }^{12,21}$ Another concern about consolidation use is whether a bone marrow in the process of recovering and regenerating from the effects of recent myelotoxic chemotherapy can sustain safely the effect of RIT. Completed studies seem to indicate that an interval period of 4 to 6 weeks post-chemotherapy is sufficient for safe administration of RIT. The inclusion of the anti-CD20 antibody in the induction chemotherapy does not eliminate the benefit of anti-CD20-based RIT. The interaction of the antibody with its target should be viewed as a dynamic process of equilibrium with constant detachment of antibody molecules and replacement by others. In any case, RIT is known to be active even in the presence of measurable rituximab levels, as shown in the study in rituximab-refractory follicular B-cell lymphoma patients. ${ }^{10}$

\section{Consolidation studies Indolent NHL}

The proof of principle of radioimmunotherapy consolidation was first demonstrated by the Southwest Oncology Group (SWOG) phase II study in 90 patients with untreated follicular B-cell non-Hodgkin's lymphoma. ${ }^{22}$ After an initial full course CHOP (cyclophosphamide, doxorubicin, vincristin, prednisolone) chemotherapy, responding patients received the radioconjugated anti-CD20 antibody ${ }^{131}$ I-tositumomab $\left.{ }^{(131} \mathrm{I}-\mathrm{T}\right)$ as consolidation. The mean time between the end of chemotherapy and the treatment with RIT was 35 days. RIT was well tolerated without excessive myelotoxicity, and $57 \%$ of the patients achieving less than a CR after chemotherapy, improved their remission with RIT. Thus the overall response rate was $90 \%$ including $67 \%$ complete responses, and the 2-year progression-free survival was estimated at $81 \%$. The same radioimmunoconjugate was tested after an abbreviated 3-cycle course of fludarabine first-line treatment. ${ }^{23}$ The sequence induced a complete response in $83 \%$ of the 35 evaluable patients. Grade 4 neutropenia or thrombocytopenia was noted in $34 \%$ and $29 \%$ respectively.

A number of studies have subsequently examined the role of ${ }^{90} \mathrm{Y}$-IT as consolidation treatment in B-cell NHL. In the Sarah Cannon Cancer Center, an abbreviated 3-cycle regimen of CHOP-rituximab or CVP (cyclophosphamide, vincristine, prednisone)-rituximab was followed by ${ }^{90} \mathrm{Y}$-IT, which is thus used as a chemotherapy sparing agent. ${ }^{2490} \mathrm{Y}$-IT was given 5 to 7 weeks after the last chemotherapy cycle. All of the 22 reported patients resonded; there were 13 partial responders to chemotherapy, 10 of whom achieved a CR after ${ }^{90} \mathrm{Y}$-IT, for an overall CR rate of $86 \%$. Responses were longlasting. Limited grade 4 neutropenia or thrombocytopenia was seen ( $18 \%$ and $0 \%$ respectively). This study preliminarily addressed the relevant question of whether ${ }^{90} \mathrm{Y}$-IT consolidation would perform well after rituximab-containing induction regimens. Further evidence is provided by a phase 2 study of 3-cycle rituximab-CHOP induction chemotherapy given to patients with symptomatic or bulky untreated follicular NHL followed by ${ }^{90} \mathrm{Y}$-IT consolidation supplemented by 4 additional rituximab infusions. ${ }^{25}$ In that study involving 60 patients, the addition of RIT consolidation nearly doubled the PET-confirmed CR rate achieved by rituximab-CHOP, from $46 \%$ to $89 \%$. After a median follow up of 19 months, 10 patients relapsed, an outcome better than expected.

Results of ${ }^{90} \mathrm{Y}$-IT consolidation following alternative chemotherapy (fludarabine-mitoxantrone combination) are suggestive of similar efficacy. Applying ${ }^{90} \mathrm{Y}$-IT consolidation to a group of 61 patients with follicular NHL after treatment with 6 cycles of oral fludarabine and mitoxantrone resulted in a $96 \%$ overall response rate. ${ }^{26}$ Radioimmunotherapy converted 12 of the observed partial responses to complete ones. With a median observation time of 30 months, the 3 -year actuarial progression-free and overall survival was $76 \%$ and $100 \%$ respectively. Grade 3 or 4 hematologic toxicity was noted in 36 of 57 evaluable patients, and blood or platelet transfusions were often required. The feasibility of the same strategy with analogous results has been reported by the same group in patients with non-follicular 
indolent B-cell lymphoma. ${ }^{27}$ Although direct comparisons are not possible, it is likely that the type of regimen as well as the number of chemotherapy cycles may have resulted in increased hematologic toxicity compared to the Sarah Cannon study.

\section{Phase 3 study of ${ }^{90} \mathrm{Y}-\mathrm{IT}$ consolidation}

The remarkable and consistent observation across these phase 2 studies of the capacity of ${ }^{90} \mathrm{Y}$-IT to further enhance the response obtained by chemotherapy led the design of a multicenter phase 3 study known with the acronym FIT. ${ }^{28}$ In order to overcome difference of opinions among various lymphoma specialists regarding the optimal first line regimen, the study randomized 409 patients with stage III and IV follicular NHL after they had achieved a response to a firstline induction regimen of the choice of the site investigators. Patients were assigned to either receive ${ }^{90} \mathrm{Y}$-IT consolidation or just be observed, with progression-free survival being the primary end-point. Nearly all patients had stage 3 or 4 disease and were roughly equally divided between partial and complete responders. The majority of patients had received alkylator-based regimens, predominately CHOP. Fludarabine combination was given to $5.4 \%$ and $15 \%$ had been exposed to rituximab.

The administration of ${ }^{90} \mathrm{Y}$-IT, as expected, increased the complete response rate, which at the end of the treatment was $87 \%$ in the radioimmunotherapy group and 53\% in the control group. This resulted in a remarkable prolongation of the progression-free survival from a median of 13.5 months in the control group to 37 months $(P<0.0001)$. In the subset of patients with partial response who entered the randomization, the difference was even more pronounced (6.3 months vs 29.7 months, $P<0.0001)$. The benefit in complete response and prolongation of the time to treatment failure was seen in all treatment groups, regardless of the intensity of the induction regimen. However, because of the small size of the subset of patients who had received rituximab-based treatment, the difference in this group did not reach statistical significance (CR rate of $71 \%$ vs $93 \%$, overlapping 95\% confidence intervals). Overall survival data are premature, but given the likelihood of crossover and multiple lines of subsequent treatments, it is doubtful that an improvent in overall survival can be demonstrated from this study. Nevertheless, updated results confirm the long term safety of RIT consolidation, as well as the feasibility of subsequent treatments, as expected. ${ }^{29}$ The rates of grade 3-4 thrombocytopenia, neutropenia and anemia in the ${ }^{90} \mathrm{Y}$-IT group was $61 \%, 67 \%$ and $3 \%$ respectively. Despite that, grade 4 infections were observed in only $8 \%$ of the patients. Platelet transfusions were given at the discretion of the investigators to 42 patients (20\%), and leukocyte growth factor support to 36 patients.

This randomized study confirmed the value of radioimmunotherapy given as supplement to chemotherapy and has led to the approval of ${ }^{90} \mathrm{Y}$-IT as consolidation after induction chemotherapy for follicular lymphoma. However this study leaves several unanswered questions. For instance, it leaves unsettled the question of whether reserving radioimmunotherapy delivery as treatment upon progression would benefit the patient at the same extent. On the other hand it is possible that the temporal proximity of radioimmunotherapy to lymphoma cells recently exposed to chemotherapy may make them more susceptible to apoptosis and thus enhance cell killing conferred by irradiation (chemosensitization). Another somewhat unclear issue is the exact magnitude of benefit from ${ }^{90} \mathrm{Y}$-IT consolidation after rituximab-containing induction chemotherapy. Along those lines, as rituximab maintenance is gaining acceptance, a subsequent multicenter randomized study is underway, comparing RIT consolidation to rituximab mainenance, or assessing the benefit of the addition of ${ }^{90} \mathrm{Y}$-IT consolidation on chemoimmunotherapy followed by rituximab maintenance backbone. It is noteworthy that the magnitude of the benefit of ${ }^{90} \mathrm{Y}$-IT consolidation seems to be in the same range of that conferred by rituximab maintenance; for the sake of comparison, adding 4 weekly rituximab infusions every 6 months for 2 years after CVP-rituximab induction chemotherapy for follicular lymphoma increased the 3-year disease-free survival from $33 \%$ to $68 \% .^{30}$ Notwithstanding the above reservations, the FIT trial has demonstrated beyond doubt the value of radioimmunotherapy consolidation in the first-line treatment of patients with follicular indolent lymphoma.

\section{${ }^{90}$ Y-IT consolidation use for aggressive $\mathrm{NHL}$}

The documentation of considerable activity of ${ }^{90} \mathrm{Y}$-IT in patients with relapsed or refractory diffuse large B-cell lymphoma ${ }^{10,15}$ as well as the desire for further improvement of the overall survival in such group of patients led to the initiation of consolidation studies, most of which are still in progress. A phase 2 study describing the administration of ${ }^{90} \mathrm{Y}$-IT as consolidation after full course of CHOP chemotherapy in patients over the age of 60 has been published and documents again the feasibility of this strategy, resulting in a $100 \%$ overall response rate and an actuarial 2-year disease-free survival of 2 years. ${ }^{31}$ Ongoing studies aim at reproducing these results. Consolidation with ${ }^{90} \mathrm{Y}$-IT has also 
been studied in patients with stage I-II diffuse large B-cell lymphoma of relatively high risk who were treated with $\mathrm{CHOP}$ for 3 cycles and involved field irradiation; ${ }^{90} \mathrm{Y}$-IT consolidation was added to this standard regimen. ${ }^{32}$ The 2 -year disease-free survival was estimated to be $91 \%$, an encouraging outcome. A randomized study assessing the role of Zevalin ${ }^{\circledR}$ as consolidation after completion of full course R-CHOP in patients with aggressive lymphoma is underway. The goal of treatment of patients with aggressive lymphoma is to produce cures and, albeit somewhat ambitiously, such studies intend to explore a possible increase in overall survival.

In mantle cell lymphoma, the sequence CHOP-rituximab followed by ${ }^{90} \mathrm{Y}$-IT consolidation has been tested as first-line treatment in 56 patients by the Eastern Cooperative Oncology Group (ECOG). ${ }^{33}$ After 4 cycles of R-CHOP, the overall response rate and the complete response rate were $72 \%$ and $14 \%$ respectively. Similar to other consolidation studies, after administration of ${ }^{90} \mathrm{Y}$-IT the responses increased respectively to $84 \%$ and $45 \%$. A similar approach employing the combination of fludarabine, mitoxantrone, cyclophosphamide and rituximab (FCM-R) as induction regimen followed by ${ }^{90} \mathrm{Y}$-IT consolidation was reported to induce CR in 19 out of 20 patients with untreated mantle cell lymphoma and with an excellent so far freedom from progression. ${ }^{34}$ However, for relapsed mantle cell lymphoma, the same regimen was less efficacious; it induced a $40 \% \mathrm{CR}$ and a TTP of 7 months in a small group of patients. Similar strategies are pursued by other groups. It emerges from the above studies, that ${ }^{90} \mathrm{Y}$-IT may be particularly helpful in the treatment of mantle cell lymphoma as consolidation after first-line chemotherapy, but its usefulness in relapsed disease, either as monotherapy or as consolidation is limited.

\section{Conclusion}

The clinical evidence discussed above supports the role of ${ }^{90} \mathrm{Y}$-IT as a useful consolidation agent after induction chemotherapy for lymphoma. In particular, the randomized phase 3 study (FIT) has demonstrated a remarkable prolongation of time to disease progression conferred by ${ }^{90} \mathrm{Y}$-IT consolidation after induction chemotherapy in patients with follicular non-Hodgkin's B-cell lymphoma and therefore has produced an unequivocal clinical paradigm. This use can be supported by the feasibility of subsequent chemotherapy treatments, the apparent lack of long-term toxicity and the low infectious risk. Such application should be considered in patients with follicular lymphoma when prolongation of remission is desired. This concept is particularly useful for patients with less than complete response to chemotherapy.
The clinical merits of such consolidation use should be carefully weighed in each individual patient case taking into account the goals of treatment and the willingness to incur the risk of radioimmunotherapy-related toxicity. Whether the pre-emptive treatment with RIT of the residual disease may be more beneficial in terms of overall survival compared to reserving RIT for the inevitable progression of the indolent lymphoma will remain an important question to be answered in the subsequent years. If the hypothesis of "temporal synergy", as hypothesized above, is true, then consolidation use ought to be more beneficial than RIT treatment upon progression.

The conclusions of the FIT study allow the incorporation of RIT consolidation in an overall treatment strategy for follicular lymphoma, that may also include protracted maintenance such as ritiximab long-term use. Accordingly, it is conceivable that a successful future strategy for follicular NHL may include an induction chemotherapy regimen followed by ${ }^{90} \mathrm{Y}$-IT consolidation, to be followed by rituximab maintenance; this remains an intriguing research question for the future. Presently, radioimmunotherapy consolidation with ${ }^{90} \mathrm{Y}$-IT represents an attractive and relatively brief consolidation treatment option, associated with prolongation of remission, a particularly desired outcome for most patients with follicular lymphoma. Ongoing randomized studies may further elucidate its role as consolidation agent for aggressive lymphoma and within the framework of an ever-changing lymphoma standard of care.

\section{Disclosure}

The author declares no conflicts of interest.

\section{References}

1. Illidge T, Chan C. How have outcomes for patients with follicular lymphoma changed with the addition of monoclonal antibodies? Leuk Lymphoma. 2008;49:1263-1273.

2. Emmanouilides C. Radioimmunotherapy for non-hodgkin lymphoma: historical perspective and current status. J Clin Exp Hematop. 2007;47:43-60.

3. Karagiannis TC. Radioimmunotherapy: Principles, current trends and future directions. Hell J Nucl Med. 2004;7:39-43.

4. Gordon LI, Molina A, Witzig T, et al. Durable responses after ibritumomab tiuxetan radioimmunotherapy for CD20+ B-cell lymphoma: long-term follow-up of a phase $1 / 2$ study. Blood. 2004;103:4429-4431.

5. Silverman DH, Delpassand ES, Torabi F, Goy A, McLaughlin P, Murray JL. Radiolabeled antibody therapy in non-Hodgkins lymphoma: radiation protection, isotope comparisons and quality of life issues. Cancer Treat Rev. 2004;30:165-172.

6. Wiseman GA, Witzig TE. Yttrium-90 $\left({ }^{90} \mathrm{Y}\right)$ ibritumomab tiuxetan (Zevalin) induces long-term durable responses in patients with relapsed or refractory B-Cell non-Hodgkin's lymphoma. Cancer Biother Radiopharm. 2005;20:185-188. 
7. Wiseman GA, Leigh B, Erwin WD, et al. Radiation dosimetry results for Zevalin radioimmunotherapy of rituximab-refractory non-Hodgkin lymphoma. Cancer. 2002;94:1349-1357.

8. Wiseman GA, Kornmehl E, Leigh B, et al. Radiation dosimetry results and safety correlations from ${ }^{90} \mathrm{Y}$-ibritumomab tiuxetan radioimmunotherapy for relapsed or refractory non-Hodgkin's lymphoma: combined data from 4 clinical trials. J Nucl Med. 2003;44:465-474.

9. Otte A. Diagnostic imaging prior to ${ }^{90} \mathrm{Y}$-ibritumomab tiuxetan (Zevalin) treatment in follicular non-Hodgkin's lymphoma. Hell J Nucl Med. 2008;11:12-15.

10. Witzig TE, White CA, Wiseman GA, et al. Phase I/II trial of IDEC-Y2B8 radioimmunotherapy for treatment of relapsed or refractory CD20(+) B-cell non-Hodgkin's lymphoma. J Clin Oncol. 1999; 17:3793-3803.

11. Emmanouilides C. Radioimmunotherapy for non-Hodgkin's lymphoma. Semin Oncol. 2003;30:531-544.

12. Witzig TE, Flinn IW, Gordon LI, et al. Treatment with ibritumomab tiuxetan radioimmunotherapy in patients with rituximab-refractory follicular non-Hodgkin's lymphoma. J Clin Oncol. 2002;20: 3262-3269.

13. Witzig TE, Gordon LI, Cabanillas F, et al. Randomized controlled trial of yttrium-90-labeled ibritumomab tiuxetan radioimmunotherapy versus rituximab immunotherapy for patients with relapsed or refractory low-grade, follicular, or transformed B-cell non-Hodgkin's lymphoma. J Clin Oncol. 2002;20:2453-2463.

14. Emmanouilides C, Witzig TE, Wiseman GA, et al. Safety and efficacy of yttrium-90 ibritumomab tiuxetan in older patients with non-Hodgkin's lymphoma. Cancer Biother Radiopharm. 2007;22:684-691.

15. Morschhauser F, Illidge T, Huglo D, et al. Efficacy and safety of yttrium-90 ibritumomab tiuxetan in patients with relapsed or refractory diffuse large B-cell lymphoma not appropriate for autologous stem-cell transplantation. Blood. 2007;110:54-58.

16. Wang $\mathrm{M}$, et al. A Phase II study of yttrium $90\left({ }^{90} \mathrm{Y}\right)$ ibritumomab tiuxetan (Zevalin $\left.{ }^{\circledR}\right)$ in patients with relapsed and refractory mantle cell lymphoma (MCL). Blood. 2006;108:767a.

17. Emmanouilides C, Witzig TE, Gordon LI, et al. Treatment with yttrium 90 ibritumomab tiuxetan at early relapse is safe and effective in patients with previously treated B-cell non-Hodgkin's lymphoma. Leuk Lymphoma. 2006;47:629-636.

18. Press OW. Evidence mounts for the efficacy of radioimmunotherapy for B-cell lymphomas. J Clin Oncol. 2008;26:5147-5150.

19. Czuczman MS, Emmanouilides C, Darif M, et al. Treatment-related myelodysplastic syndrome and acute myelogenous leukemia in patients treated with ibritumomab tiuxetan radioimmunotherapy. J Clin Oncol. 2007;25:4285-4292.

20. Ansell SM, Schilder RJ, Pieslor PC, et al. Antilymphoma treatments given subsequent to yttrium 90 ibritumomab tiuxetan are feasible in patients with progressive non-Hodgkin's lymphoma: a review of the literature. Clin Lymphoma. 2004;5:202-204.

21. Kaminski MS, Zelenetz AD, Press OW, et al. Pivotal study of iodine I 131 tositumomab for chemotherapy-refractory low-grade or transformed low-grade B-cell non-Hodgkin's lymphomas. J Clin Oncol. 2001;19:3918-3928.
22. Press OW, Unger JM, Braziel RM, et al. A phase 2 trial of CHOP chemotherapy followed by tositumomab/iodine I 131 tositumomab for previously untreated follicular non-Hodgkin lymphoma: Southwest Oncology Group Protocol S9911. Blood. 2003;102:1606-1612.

23. Leonard JP, Coleman M, Kostakoglu L, et al. Abbreviated chemotherapy with fludarabine followed by tositumomab and iodine I 131 tositumomab for untreated follicular lymphoma. J Clin Oncol. 2005;23:5696-5704.

24. Shipley DL, Spigel DR, Carrell DL. Phase II trial of rituximab and short duration chemotherapy followed by ${ }^{90} \mathrm{Y}$-ibritumomab tiuxetan as first line treatment for patients with follicular lymphoma: A Minnie Pearl Cancer Network phase II trial. J Clin Oncol. 2004;22:6519a.

25. Jacobs SA, Swerdlow SH, Kant J, et al. Phase II trial of short-course CHOP-R followed by ${ }^{90} \mathrm{Y}$-ibritumomab tiuxetan and extended rituximab in previously untreated follicular lymphoma. Clin Cancer Res. 2008; 14:7088-7094.

26. Zinzani PL, Tani M, Pulsoni A, et al. Fludarabine and mitoxantrone followed by yttrium-90 ibritumomab tiuxetan in previously untreated patients with follicular non-Hodgkin lymphoma trial: a phase II nonrandomised trial (FLUMIZ). Lancet Oncol. 2008;9:352-358.

27. Zinzani PL, Tani M, Fanti S, et al. A phase 2 trial of fludarabine and mitoxantrone chemotherapy followed by yttrium-90 ibritumomab tiuxetan for patients with previously untreated, indolent, nonfollicular, non-Hodgkin lymphoma. Cancer. 2008;112:856-862.

28. Morschhauser F, Radford J, Van Hoof A, et al. Phase III trial of consolidation therapy with yttrium-90-ibritumomab tiuxetan compared with no additional therapy after first remission in advanced follicular lymphoma. J Clin Oncol. 2008;26:5156-5164.

29. Morschhauser F, et al. Extended follow-up of the International Randomized Phase 3 First-Line Indolent Trial (FIT) shows durable benefit of ${ }^{90} \mathrm{Y}$-ibritumomab tiuxetan $\left(\right.$ Zevalin $\left.^{\circledR}\right)$ consolidation of first remission in advanced stage follicular non-Hodgkin's Lymphoma. Blood. 2008;112:202.

30. Hochster H, Weller E, Gascoyne RD, et al. Maintenance rituximab after cyclophosphamide, vincristine, and prednisone prolongs progressionfree survival in advanced indolent lymphoma: results of the randomized Phase III ECOG1496 Study. J Clin Oncol. 2009;27:1607-1614.

31. Zinzani PL, Tani M, Fanti S, et al. A phase II trial of CHOP chemotherapy followed by yttrium 90 ibritumomab tiuxetan (Zevalin) for previously untreated elderly diffuse large B-cell lymphoma patients. Ann Oncol. 2008;19:769-773.

32. Thomas P, Miller, et al. Effect of adding ibritumomab tiuxetan (zevalin) radioimmunotherapy consolidation to three Cycles of CHOP plus involved-field radiotherapy for limited-stage aggressive diffuse B-cell lymphoma (SWOG 0313). Blood. 2008;112:3598.

33. Smith MR, et al. Phase II study of rituximab + CHOP followed by ${ }^{90} \mathrm{Y}$-ibritumomab tiuxetan in patients with previously untreated mantle cell lymphoma: An Eastern Cooperative Oncology Group Study (E1499). J Clin Oncol. 2006; ASCO Meeting Abstracts Jun 20: a7503.

34. Jurczak W, et al. ${ }^{90} \mathrm{Y}-\mathrm{Z}$ evalin ${ }^{\circledR}\left({ }^{90} \mathrm{Y}\right.$-ibritumomab tiuxetan $)$ radioimmunotherapy (RIT) consolidation of FCM induction chemotherapy in mantle cell lymphoma (MCL) patients: Results from the PLRG upon completed enrollment. Blood. 2006;108 (Suppl 1):777a.

Cancer Management and Research

\section{Publish your work in this journal}

Cancer Management and Research is an international, peer-reviewed open access journal focusing on cancer research and the optimal use of preventative and integrated treatment interventions to achieve improved outcomes, enhanced survival and quality of life for the cancer patient. The journal welcomes original research, clinical \& epidemiological

studies, reviews \& evaluations, guidelines, expert opinion \& commentary, case reports \& extended reports. The manuscript management system is completely online and includes a very quick and fair peerreview system, which is all easy to use. Visit http://www.dovepress.com/ testimonials.php to read real quotes from published authors.

\section{Dovepress}

